\title{
Peroxynitrite Decomposition Catalyst FeTMPyP Provides Partial Protection Against Intestinal Ischemia and Reperfusion Injury in Infant Rats
}

\author{
GIORGIO STEFANUTTI, AGOSTINO PIERRO, VIRPI V. SMITH, NIGEL J. KLEIN AND SIMON EATON \\ Units of Paediatric Surgery [G.S., A.P., S.E.], Gastroenterology and Autoimmunity [V.V.S], and Immunobiology [N.J.K.], Institute of Child \\ Health, London, WC1N 1EH, United Kingdom
}

\begin{abstract}
Free radicals are important in development of intestinal ischemia-reperfusion (I/R) injury, leading to intestinal and pulmonary damage. We evaluated the effects of peroxynitrite decomposition catalyst FeTMPyP in infant intestinal I/R. Suckling rats underwent 40min intestinal ischemia $+90 \mathrm{~min}$ reperfusion. At reperfusion, animals received saline or FeTMPyP. Groups were $(n=$ 11 per group): 1) control+saline; 2) I/R + saline; 3) I/R+FeTMPyP. Increased histologic injury and ICAM-1 expression were observed in ileum of both $\mathrm{I} / \mathrm{R}+$ saline and $\mathrm{I} / \mathrm{R}+\mathrm{FeTMPyP}$ rats, but P-selectin expression was increased in $\mathrm{I} / \mathrm{R}+$ saline animals only versus controls. Myeloperoxidase (neutrophil infiltration marker) was increased in ileum and lungs of I/R + saline rats, but FeTMPyP prevented this in the ileum. I/R+ saline animals showed higher malondialdehyde (lipid peroxidation marker) in ileum and lungs versus both control+saline and I/R + FeTMPyP rats. Glutathione was decreased in all I/R animals, but oxidized and total glutathione were higher in $\mathrm{I} / \mathrm{R}+\mathrm{FeTMPyP}$ than the $\mathrm{I} / \mathrm{R}+$ saline group. Nitrate + nitrite concentration (systemic nitric oxide production) was elevated in $\mathrm{I} / \mathrm{R}+$ saline but not in I/R+FeTMPyP animals. FeTMPyP provides limited protection against intestinal $\mathrm{I} / \mathrm{R}$ in neonatal rats by reducing ileal $\mathrm{P}$-selectin expression, systemic nitric oxide production, neutrophil infiltration in ileum and lipid peroxidation in both lungs and ileum; and preserving intestinal antioxidant capacity. (Pediatr Res 62: 43-48, 2007)
\end{abstract}

$\mathrm{I}^{\mathrm{n}}$ ntestinal ischemia and reperfusion (I/R) is an important component of several life- threatening conditions in adults (1). In addition, infants are at risk from the consequences of intestinal $\mathrm{I} / \mathrm{R}$, which is involved in the pathogenesis of necrotizing enterocolitis (NEC) (2), can cause intestinal necrosis following midgut volvulus (3) or intussusception (4), and result from splanchnic hypoperfusion during sepsis (5) or after hemorrhage (6). Multiple organ dysfunction syndrome (MODS) is a well-recognized complication of pediatric conditions associated with intestinal I/R injury and respiratory failure has been shown to be a crucial determinant of early mortality (7).

Although there is little work concerning intestinal I/R injury in developing animals, studies in adult animal models have shown that development of an inflammatory response plays a pivotal role in the pathogenesis of intestinal and distal organ injury $(8,9)$. In particular, infiltration of neutrophils and their

Received October 20, 2006; accepted February 26, 2007.

Correspondence: Simon Eaton, Ph.D., Department of Paediatric Surgery, Institute of Child Health, 30, Guilford Street, London WC1N 1EH; e-mail: s.eaton@ich.ucl.ac.uk This work was supported by grants from Fondazione Eugenio Litta and SPARKS. subsequent action is believed to be responsible for tissue damage within the intestine following intestinal I/R $(10,11)$, and also in distant organs, such as lungs, liver and heart (12), and we have recently shown that neutrophil infiltration correlates with histologic damage in human NEC (13), although other mechanisms may also be important in intestinal $\mathrm{I} / \mathrm{R}$ injury $(14,15)$. Leukocyte-endothelial cell interaction represents a crucial step in migration of leukocytes into tissues, which is mediated by several adhesion molecules expressed by activated endothelium (including P-selectin, intercellular cell adhesion molecule-1 [ICAM-1] and vascular cell adhesion molecule-1 [VCAM-1]).

Tissue injury occurs following neutrophil infiltration as a consequence of the release of a wide array of enzymes by activated neutrophils. In addition, production of reactive oxygen species by activated neutrophils, from xanthine oxidase and other intracellular sources, can lead to oxidative stress once the scavenging potential of endogenous antioxidant systems is overwhelmed, resulting in protein modification, lipid peroxidation and DNA damage.

Of the variety of reactive oxygen and nitrogen species generated during oxidative stress, peroxynitrite is one of the most reactive. It is produced by spontaneous combination of NO and superoxide released during reperfusion (16). Peroxynitrite is responsible for a significant proportion of deleterious effects previously attributed to NO or superoxide alone, including DNA strand scission (17), lipid peroxidation (18) and enzyme inactivation $(19,20)$. Peroxynitrite decomposition catalysts are a class of porphyrin-containing compounds catalysing decomposition of peroxynitrite to the nonradical nitrate. In particular, 5,10,15,20-tetrakis ( $N$-methyl-4'pyridyl) porphyrinato iron (III) (FeTMPyP) has been shown to effectively reduce intestinal injury in experimental endotoxemia (21), and to improve cerebral I/R injury (22). Part of the protective effect of FeTMPyP may be exerted via interruption of the inflammatory cascade by preventing activation of NFkappa-B (23). A similar compound, 5,10,15,20-tetrakis (2,4,6trimethyl-3, 5-disulfonatophenyl)-porphyrinato iron (III)

Abbreviations: I/R, ischemia and reperfusion; MPO, myeloperoxidase; MDA, malondialdehyde; NEC, necrotizing enterocolitis; ICAM, intercellular cell adhesion molecule-1; VCAM, vascular cell adhesion molecule-1 
(FeTMPS) has been shown to reduce local injury following intestinal $\mathrm{I} / \mathrm{R}$ in adult rats (8).

The aim of this study was to characterize intestinal and pulmonary injury in an infant rat model of intestinal I/R, and to evaluate the effects of FeTMPyP as a possible therapeutic agent.

\section{METHODS}

Experimental groups. The study was approved under the United Kingdom Home Office Animals (Scientific Procedures) Act 1986. Suckling rats (11-13 d old, $20-30 \mathrm{~g}$ ) were randomly divided into three groups ( $n=11$ per group): i) control for 130-min with saline infusion; ii) 40-min intestinal ischemia, followed by 90 -min reperfusion with saline infusion; iii) 40-min intestinal ischemia, followed by 90 -min reperfusion with FeTMPyP infusion.

Surgical procedures. Animals were anesthetized by inhalation of oxygen/ nitrous oxide with $1.5 \%$ to $2.0 \%$ isoflurane via a nose cone as previously described (24). Rectal temperature was monitored and was maintained within normal values for suckling rats of this age (i.e., $\left.34-36^{\circ} \mathrm{C}(25)\right)$ using a heating blanket and lamp. Surgical procedures were performed under microscopic view. Control animals underwent a transverse laparotomy with identification of the superior mesenteric artery only. In animals undergoing intestinal $I / R$, the superior mesenteric artery was occluded at the origin with a microvascular clip (S\&T AG, Neuhausen, Switzerland) and occlusion confirmed by absence of pulsation in vascular arcades of the mesentery. After $40 \mathrm{~min}$, the clip was removed and reperfusion confirmed by return of arterial pulsation in the ileum.

Fluid administration. In all animals, a $0.16 \mathrm{~mm}$ polyimide catheter (Cole-Parmer, Vernon Hills, IL) was inserted immediately after the first laparotomy in the right femoral vein for fluid infusion. Five minutes before reperfusion, rats received either $5 \mathrm{~mL} / \mathrm{kg} 0.9 \%$ saline (control group and saline plus I/R group), or $30 \mathrm{mg} / \mathrm{kg}$ FeTMPyP (Calbiochem, Nottingham, UK) in $5 \mathrm{~mL} / \mathrm{kg} 0.9 \%$ saline (FeTMPyp group) through the femoral line. One minute before the end of the experiment, all animals were infused with $1,000 \mathrm{IU} / \mathrm{kg}$ sodium heparin in $5 \mathrm{~mL} / \mathrm{kg} 0.9 \%$ saline.

Sample collection. At sacrifice, a blood sample was collected into a heparin tube and centrifuged (3000rpm for $5 \mathrm{~min}$ at $4^{\circ} \mathrm{C}$ ) and plasma stored at $-40^{\circ} \mathrm{C}$ until analysis. Small intestine $(5-20 \mathrm{~cm}$ from the ileocecal valve) and lungs were also removed and stored at $-40^{\circ} \mathrm{C}$. Terminal ileum (2 to $5 \mathrm{~cm}$ from the ileocecal valve) was fixed in $10 \%$ formaldehyde for $24 \mathrm{~h}$ and embedded in paraffin.

Histologic injury. Histologic injury was evaluated and graded blindly by three investigators on hematoxylin and eosin stained sections using a wellestablished scoring system (26): 1) Normal mucosal villi; 2) Development of mucosal slough at villous tips; 3 ) Extension of the sub-epithelial space with the epithelial layer lifting up in sheets, presence of a few denuded villous tips, and mild capillary congestion; 4) Denuded villi with exposed lamina propria, dilated, exposed capillaries with evidence of hemorrhage, and increased cellularity of the lamina propria; 5) Digestion and disintegration of the lamina propria and presence of hemorrhage and ulceration.

Expression of endothelial adhesion molecules. Immunostaining for ileal P-Selectin (CD62P), ICAM-1 (CD54) and V-CAM-1 (CD106) was performed on paraffin-embedded sections.

$P$-selectin $(C D 62 P)$. Immunostaining was carried out as previously described (13) using the same CD-62P antibody which cross reacts with both rat and human antigens (BD PharMingen, San Diego, CA).

ICAM-1. After microwave heating in $1 \%$ citrate buffer $\mathrm{pH}$ 6.0, blockade of endogenous peroxidase activity and incubation with $10 \%$ normal goat serum were performed. Primary antibody [ICAM-1 (CD54), Endogen, Woburn, MA, 1:2000] was applied for 60min at room temperature, and sections incubated with anti-mouse labeled polymer HRP and DAB (EnVision, DakoCytomation, Cambridge, UK).

$V C A M-1$.Sections underwent blockade of endogenous peroxidase activity and incubation with $10 \%$ normal goat serum and were incubated with VCAM-1 [VCAM-1 (CD106), BD PharMingen, San Diego, CA, 1:15000] for $60 \mathrm{~min}$ at room temperature. Anti-mouse labeled polymer $\mathrm{HRP}$ and $\mathrm{DAB}$ were then applied.

Expression of P-selectin, ICAM-1 and VCAM-1 was assessed by grading the staining intensity of the endothelium of subepithelial vessels. The specimens were scored blindly by three authors on a semi-quantitative scale based both on the number of positive vessels and on the intensity of the staining: 1) Weak; 2) Mild; 3) Moderate; 4) Strong.

Myeloperoxidase activity. Tissue samples (right lung and ileum $5-10 \mathrm{~cm}$ from the ileocecal valve) were homogenized using an Ultra-Turrax homoge- nizer in $2 \mathrm{~mL} 50 \mathrm{mM}$ potassium phosphate buffer (pH 6.0) containing $0.5 \%$ (wt/vol) hexadecyltrimethylammonium bromide. Myeloperoxidase (MPO) activity (marker of neutrophil infiltration) was measured in the homogenate as previously described (27), and expressed as milliunits (mU) per mg protein.

Malondialdehyde concentration. Levels of malondialdehyde MDA (a marker of lipid peroxidation) in tissues (right lung and ileum $5-10 \mathrm{~cm}$ from the ileocecal valve) were measured by HPLC as previously described (28).

Quantification of oxidized and reduced glutathione. The concentrations of reduced (GSH) and oxidized (GSSG) glutathione (a major endogenous antioxidant) in the small intestine $(0.2-0.3 \mathrm{~g}, 10$ to $20 \mathrm{~cm}$ from the ileocecal valve) were measured by HPLC as previously described (28); total glutathione was calculated as GSH + 2GSSG.

Quantification of nitrate plus nitrite. The plasma concentration of nitrate plus nitrite, which reflects systemic production of nitric oxide (NO), was measured by HPLC (28).

Statistical analysis. Continuous variables are expressed as mean \pm SEM and compared by ANOVA with Tukey posthoc test. Data referring to discrete variables are expressed as median [interquartile range], and Kruskal-Wallis test was used for group comparison. Linear regression was used to evaluate the correlation between levels of MDA and glutathione in the ileum; and Spearman-R to assess the relationships between histologic injury, P-Selectin and ICAM-1 expression, and MPO activity. Results showing $\mathrm{p}$ values $<0.05$ were considered significant.

\section{RESULTS}

Histologic injury. Control animals showed normal intestinal architecture, characterized by well-preserved mucosa with long villi (Fig. $1 A$ ). All animals undergoing intestinal I/R with saline infusion developed histologic injury, ranging from mild mucosal damage with sloughing at villous tips to complete destruction of the mucosa and the lamina propria and signs of hemorrhage (Fig. 1A). Histologic damage was
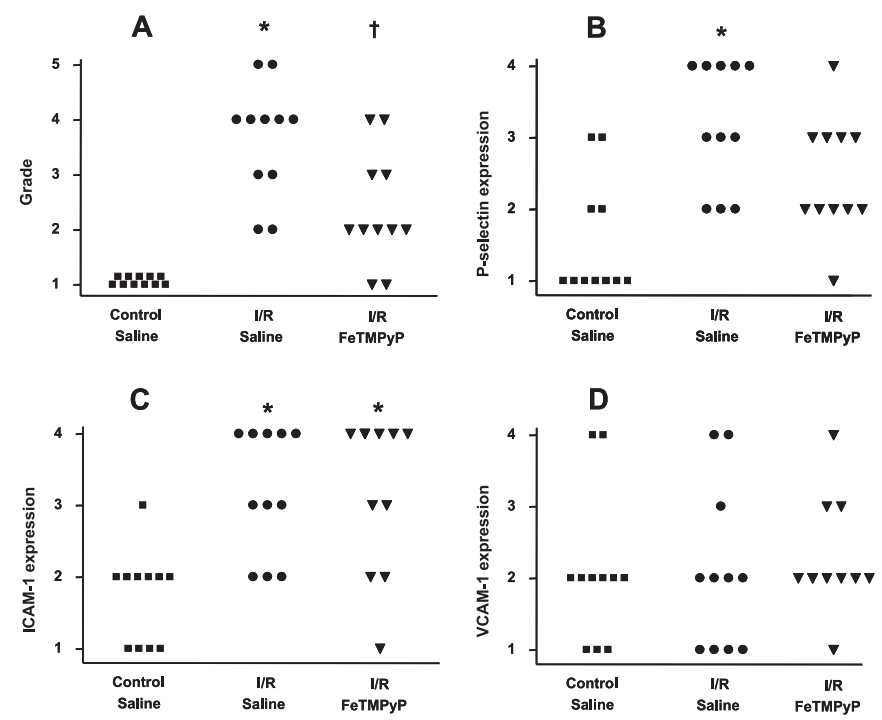

Figure 1. Histologic injury and adhesion molecule expression. Animals underwent sham operation with saline infusion (Control + Saline), or intestinal ischemia and reperfusion with infusion of either saline (I/R + Saline) or FeTMPyP solution (I/R + FeTMPyP). (A) Degree of histologic injury (Grade $1=$ normal histology; Grade $5=$ severe injury) in the ileum. $[B]$ : Degree of expression of P-selectin (Grade $1=$ weak expression; Grade $4=$ strong expression) in the ileum. $[C]$ : Degree of expression of ICAM-1 (Grade $1=$ weak expression; Grade $4=$ strong expression) in the ileum. $[D]$ : Degree of expression of VCAM-1 (Grade $1=$ weak expression; Grade 4 = strong expression) in the ileum. Data ( $n=11$ per group) are expressed as median [interquartile range] and were compared by Kruskal-Wallis test. $* p<0.001$ vs. Control + Saline, $\uparrow p<0.05 v s$. Control + Saline, $\ddagger p<0.01 v s$. Control + Saline. 


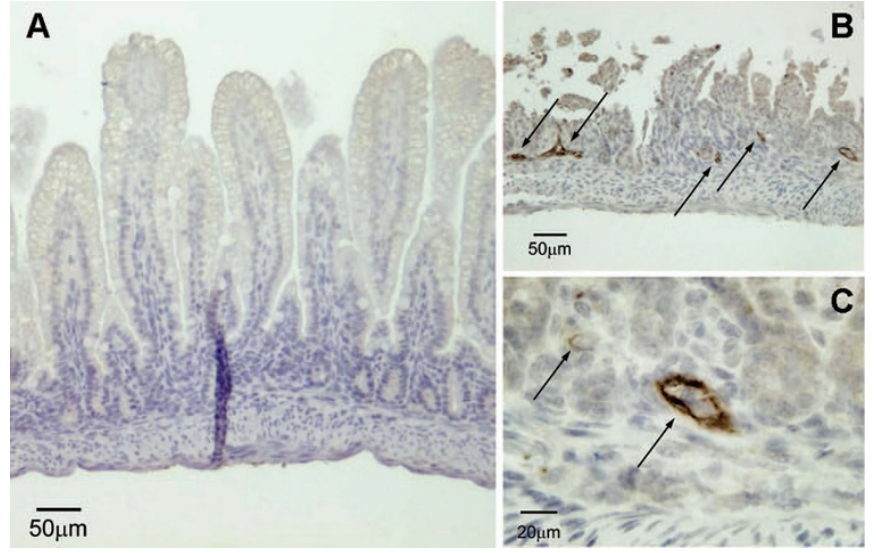

Figure 2. Expression of P-selectin (CD62P) in ileum. Animals underwent sham operation with saline infusion $(A)$, or intestinal ischemia and reperfusion with infusion of saline $(B),(C)$, and sections of ileum were immunostained for $\mathrm{P}$-selectin expression as described in the materials and methods. Expression of P-Selectin on blood vessels is indicated by the arrows. $(A),(B): \times 100$ magnification; $(C): \times 400$ magnification.

also evident on most specimens from intestinal I/R animals receiving FeTMPyP, but a trend toward improvement (even though not statistically significant) was seen in comparison to $\mathrm{I} / \mathrm{R}$ rats receiving saline alone (Fig. $1 A$ ).

Endothelial adhesion molecules. P-selectin. Expression of $\mathrm{P}$-selectin on blood vessels was weak in most specimens from control rats (Fig. 1B, Fig. 2A). Intestinal I/R induced a significant increase in the expression of P-selectin, most animals showing moderate to strong staining (Fig. 1B). Vessels expressing P-selectin were evenly distributed within the specimens, and staining appeared to be particularly intense on medium-sized vessels of the submucosa (Fig. 2B,C). However, expression of P-selectin on I/R rats receiving FeTMPyP showed only a nonsignificant trend toward increase compared with controls (Fig. 1B). A strong positive correlation was found between intensity of expression of P-selectin on blood vessels and degree of histologic injury in the ileum in rats from all experimental groups $(\mathrm{r}=0.580 ; p<0.001)$.

ICAM-1. Mild expression of ICAM-1, particularly on medium-size vessels of the submucosa, was evident in ileum of most control rats, reflecting constitutive ICAM-1 expression (Fig. 1C). However, ICAM-1 was increased following intestinal $\mathrm{I} / \mathrm{R}$ in animals receiving both saline and FeTMPyP, which appeared to show a more diffuse and intense staining on medium size vessels in the submucosa. In addition, in the latter two groups considerable ICAM- 1 was observed also on the microcirculation, particularly in the submucosa, which was virtually absent in specimens from control rats.

$V C A M-1$. VCAM-1 expression was variable in ileum from control animals (Fig. 1D). In most specimens, VCAM-1 appeared to be expressed patchily, and staining was present not only on medium-sized vessels, but also on the microcirculation in the submucosa. Rats undergoing intestinal I/R, both with and without FeTMPyP, did not show any quantitative increase in VCAM-1 expression, and the type and location of positively stained vessels was apparently similar to that of controls.
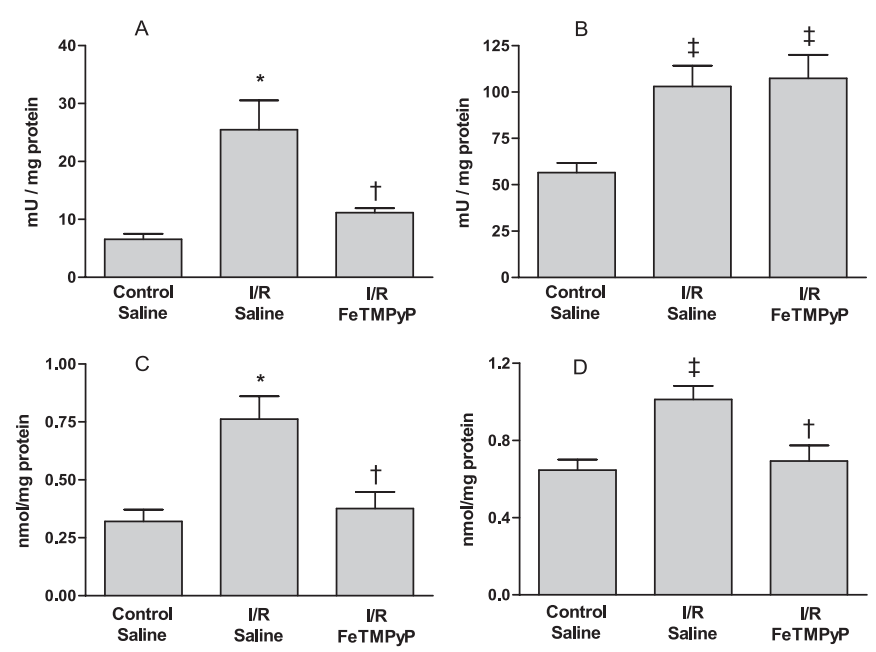

Figure 3. Myeloperoxidase (MPO) activity and malondialdehyde (MDA) concentration. Animals underwent sham operation with saline infusion (Control + Saline), or intestinal ischemia and reperfusion with infusion of either saline (I/R + Saline) or FeTMPyP solution (I/R + FeTMPyP). MPO activity was measured in the ileum $(A)$ and lungs $(B)$, and MDA concentration measured in ileum $(C)$ and lungs $(D)$. Data $(n=11$ per group) are reported as mean \pm SEM. One-way ANOVA with Tukey post hoc test was used for group comparison. ${ }^{*} p<0.001 v s$. Control + Saline, $\dagger p<0.01 v s$. I/R + Saline, $\ddagger p<0.01 v s$. Control + Saline.

Myeloperoxidase activity. Neutrophil infiltration (as assessed by MPO activity) was substantially increased in ileum of $\mathrm{I} / \mathrm{R}$ animals receiving saline compared with controls, but this effect was prevented by administration of FeTMPyP (Fig. $3 A$ ). However, neutrophil infiltration in the lungs was increased in animals from both $\mathrm{I} / \mathrm{R}+$ saline and $\mathrm{I} / \mathrm{R}+$ FeTMPyP groups (Fig. 3B).

Malondialdehyde concentration. Intestinal $\mathrm{I} / \mathrm{R}$ was also associated with the development of lipid peroxidation in both ileum and lungs, as shown by the marked increase in malondialdehyde levels of $I / R+$ saline rats with respect to the control group (Fig. 3C,D). However, no increase in malondialdehyde concentrations in either lungs or ileum was observed in $\mathrm{I} / \mathrm{R}$ animals receiving FeTMPyP compared with controls (Fig. 3C,D).

Glutathione concentration. A profound depletion in intestinal total glutathione was observed following intestinal $\mathrm{I} / \mathrm{R}$ in rats infused with saline only (Fig. $4 A$ ), which was due to a
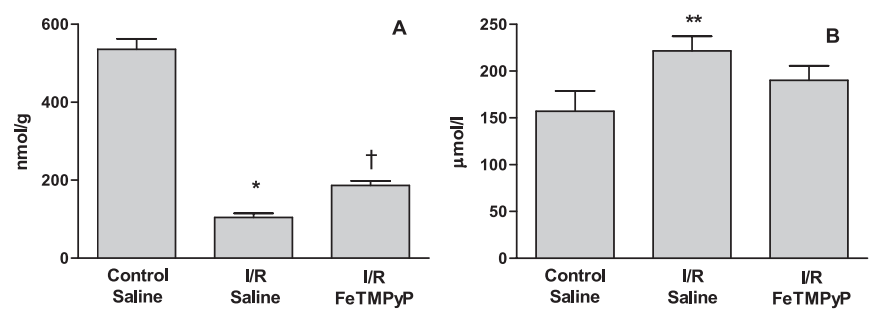

Figure 4. Glutathione and nitric oxide metabolites. Animals underwent sham operation with saline infusion (Control + Saline), or intestinal ischemia and reperfusion with infusion of either saline (I/R + Saline) or FeTMPyP solution (I/R + FeTMPyP). [A] total glutathione in ileum, [B] systemic nitric oxide production (plasma nitrate plus nitrite). Results ( $n=11$ per group) are reported as mean \pm SEM; one-way ANOVA with Tukey post hoc test was used for group comparison. $* p<0.001 v s$. Control + Saline. $\dagger p<0.001 v s$. Control + Saline, $p<0.01 v s . \mathrm{I} / \mathrm{R}+$ Saline ${ }^{*} p p<0.05 v s$. Control + Saline. 
decrease in both reduced and oxidized glutathione (results not shown). Administration of FeTMPyP partially prevented the decrease in glutathione caused by intestinal I/R (Fig. 4A). Interestingly, intestinal levels of total glutathione negatively correlated with MDA concentration $\left(\mathrm{r}^{2}=0.195 ; p=0.010\right)$, suggesting an association between a decrease in antioxidant potential and development of lipid peroxidation.

Nitrate plus nitrite concentration. Plasma concentrations of nitrate + nitrite, reflecting systemic nitric oxide production, were increased in the I/R + saline group, but not in the I/R + FeTMPyP, with respect to the control + saline group (Fig. 4B).

\section{DISCUSSION}

Ischemia-reperfusion of the intestine causes a cascade of local and distal damage and organ failure, which may be triggered by neutrophil infiltration with subsequent release of proteolytic enzymes and reactive oxygen and nitrogen species. Therapeutic strategies, which prevent local and/or distant organ damage in intestinal I/R, may exert their effects via this neutrophil-infiltration-free radical-organ damage axis. Examples are moderate hypothermia $(27,28)$, anti-adhesion molecule antibodies or other methods to deplete neutrophils (10, $11,29)$ and scavengers of reactive oxygen and nitrogen species $(8,30)$. However, many reactive oxygen and nitrogen species have important physiologic roles such as maintenance of vascular tone (nitric oxide) and bacterial killing (superoxide) so that deleterious effects could result from scavenger administration. In contrast, peroxynitrite is a reactive species with no well-defined physiologic function so could potentially be a more useful valid pharmacological target. The compound used in this study is one of a large group of transition metal based agents, which scavenge a variety of reactive nitrogen and oxygen species. One compound is in use in clinical trials for relief of dermatological pain (31). Although FeTMPyP is very specific for scavenging peroxynitrite, it appears to interrupt the cycle of adhesion molecule expression, neutrophil infiltration, antioxidant depletion, free radical production and histologic damage. This finding supports a role for peroxynitrite in initiating this cascade. Our results in the current study in infant rats, using FeTMPyP, are broadly similar to those in adult rat intestinal ischemia-reperfusion injury, using a related peroxynitrite scavenger, FeTMPS (8). There are, however, some differences between these studies, in addition to the use of infant rats and a different peroxynitrite decomposition catalyst. Cuzzocrea et al. administered the scavenger after only $15 \mathrm{~min}$ of ischemia ( $30 \mathrm{~min}$ before reperfusion), whereas we administered the scavenger $5 \mathrm{~min}$ before reperfusion (after $40 \mathrm{~min}$ of ischemia). Potential clinical use of peroxynitrite decomposition catalysts would probably involve administration close to the initiation, or even after commencement, of reperfusion (e.g., surgery for midgut volvulus). Interestingly, in a rat model of stroke, FeTMPyP was protective even when given $6 \mathrm{~h}$ after the acute insult (22), suggesting that administration could be effective even when applied later, although we did not test this in our model. We additionally showed a protective effect of FeTMPyP against lipid peroxidation in the lungs, which is important as MODS, is an important consequence of diseases involving intestinal ischemia-reperfusion injury. Although peroxynitrite scavengers decreased both ileal P-selectin expression and neutrophil infiltration in our study and that of Cuzzocrea, in their study, the peroxynitrite scavenger prevented up-regulation of ICAM expression, whereas in our study, it had no effect. Our finding suggests that prevention of P-selectin expression, and therefore of early neutrophil rolling, is sufficient to attenuate neutrophil infiltration, even when the elevated ICAM-1 expression, which mediates later firm adhesion, is unaltered. In other studies using peroxynitrite scavengers, it has been shown that removal of peroxynitrite can prevent plasma leakage into the intestinal tract in endotoxemia (21). Other similar effects have been observed using structurally-related superoxide dismutase mimetics $(21,32)$ which, by scavenging superoxide, would also prevent peroxynitrite formation, as peroxynitrite can be physiologically generated by the reaction of superoxide with nitric oxide.

Intestinal IR caused a dramatic decrease in both oxidized and reduced glutathione, which was partially prevented by FeTMPyP. Loss of glutathione in intestinal IR has been previously demonstrated $(28,33)$ and is thought to be due to a process whereby glutathione is oxidized and then is lost from cells (34), resulting in a loss of total, reduced and oxidized glutathione. Whether glutathione reacts directly with peroxynitrite or decreases peroxynitrite damage by scavenging nitric oxide is uncertain, as the reaction of glutathione with peroxynitrite is rather slow (35). An additional explanation for decreased glutathione is the increased glutathionylation of proteins during oxidative stress (36). This could have several different consequences, depending on the protein, the residue which is glutathionylated and the mechanism, which could result in a decrease in free glutathione, inhibition of protein function, or preservation of protein function if a critical cysteine residue is protected from oxidation by a reversible glutathionylation.

In our study, as FeTMPyP was administered systemically, we are unable to determine whether the effects of FeTMPyP administration on distal organs and systemic measures are secondarily mediated via the effect of FeTMPyP on the intestine, or whether FeTMPyP is also acting systemically. Interestingly, FeTMPyP decreased neutrophil infiltration and lipid peroxidation in the intestine, but lipid peroxidation in the lung was decreased despite neutrophil infiltration in the lung remaining elevated, suggesting that FeTMPyP could be acting locally in the lung to prevent peroxynitrite-initiated lipid peroxidation. However, FeTMPyP catalyses the decomposition of peroxynitrite to nitrate, so that if the effects of FeTMPyP were systemic, one could expect an increase in plasma nitrate plus nitrite, rather than the decrease we observed in the current study. The observed decrease suggests that FeTMPyP may be primarily effective locally in the intestine, thus preventing a systemic inflammatory response. Further experiments would be required to determine the mechanism of action. Although FeTMpyP significantly decreased ileal neutrophil infiltration and lipid peroxidation, and main- 
tained ileal glutathione levels, it was ineffective in decreasing histologic injury. This apparent disparity between local and distal effects could also be related to different mechanisms of damage. Although some studies have supported the importance of neutrophil-mediated local damage in intestinal IR injury (10), other mechanisms, in particular complement activation (14,37), may be more important. Recent studies suggest that activation of the classical pathway of complement is initiated by deposition of self-reactive natural $\operatorname{IgM}$ antibodies in the subepithelial space, i.e., where tissue injury is most severe (14). However, migration of neutrophils mostly occurs at a deeper site in the submucosa, which is supported by our findings of an intense adhesion molecule expression in this area. This could explain the lack of protection by FeTMPyP on intestinal tissue injury even though neutrophil sequestration was completely prevented. Our model represents severe $\mathrm{I} / \mathrm{R}$ damage to the whole intestine. It is possible that the limited effects of FeTMPyP could be beneficial to preserve areas of marginal viability, e.g., some distance away from a perforation. This would need to be the subject of further investigations.

We attempted to quantify nitrotyrosine to confirm that FeTMPyP was effective in its role as a peroxynitrite scavenger; unfortunately we were unable to obtain reliable results using a commercially available ELISA kit for nitrotyrosine. Nevertheless, we believe that the partial protection achieved in our model is most likely due to the effects of FeTMPyP in reducing peroxynitrite-mediated damage, as FeTMPyP is thought to be very specific in its action $(16,21)$.

Various compounds of this class of porphyrin derivatives have been synthesized, and a closely-related compound, M-40403, which is specific for superoxide, is currently in phase II clinical trials for inflammatory conditions and pain (31). M-40403 contains $\mathrm{Mn}$ rather than $\mathrm{Fe}$ at the catalytic centre, as metabolism of Fe-containing porphyrins could potentially lead to the generation of harmful Fe-centered radicals. However, all the metal-centered porphyrin compounds are very stable and there are no reports of prooxidant activities in vivo. A further potential problem with antioxidant therapy is interference with the bacterial killing process and increased susceptibility to infection. Peroxynitrite is toxic to bacteria and may be involved with bacterial killing following phagocytosis (38). In addition, it may have other physiologic roles (39), so that scavenging peroxynitrite may have other effects in addition to prevention of the inflammatory process.

Peroxynitrite decomposition scavengers have been shown to be effective in a variety of models, and are promising candidates for clinical trials to prevent systemic inflammatory response syndrome, especially given the apparently relative broad window of opportunity for their use. Although FeTMPyP only afforded limited protection in our model, it warrants further investigation because it is not expected to decrease bacterial killing. However, careful clinical studies are required to determine whether there is increased susceptibility to infection and/or other side effects.

\section{REFERENCES}

1. Schoenberg MH, Beger HG 1993 Reperfusion injury after intestinal ischemia. Crit Care Med 21:1376-1386

2. Eaton S, Hall N, Rees CM, Pierro A 2004 Necrotizing enterocolitis: pathogenesis, medical management and surgery. Br J Intensive Care 14:101-105

3. Clark LA. 2000 Malrotation. In: Ashcraft KW (ed) Pediatric Surgery. W.B. Saunders, Philadelphia, PA, pp 425-434.

4. Fallat ME. 2000 Intussusception. In: Ashcraft KW (ed) Pediatric Surgery. W.B. Saunders, Philadelphia, PA, pp 518-526.

5. Fink MP 1993 Adequacy of Gut Oxygenation in Endotoxemia and Sepsis. Crit Care Med 21:S4-S8

6. Moore FA 1999 The role of the gastrointestinal tract in postinjury multiple organ failure. Am J Surg 178:449-453

7. Sonntag J, Wagner MH, Waldschmidt J, Wit J, Obladen M 1998 Multisystem organ failure and capillary leak syndrome in severe necrotizing enterocolitis of very low birth weight infants. J Pediatr Surg 33:481-484

8. Cuzzocrea S, Misko TP, Costantino G, Mazzon E, Micali A, Caputi AP, MacArthur H, Salvemini D 2000 Beneficial effects of peroxynitrite decomposition catalyst in a rat model of splanchnic artery occlusion and reperfusion. FASEB J 14:1061-1072

9. Chung DH, Ethridge RT, Kim S, Owens-Stovall S, Hernandez A, Kelly DR, Evers BM 2001 Molecular mechanisms contributing to necrotizing enterocolitis. Ann Surg 233:835-842

10. Sisley AC, Desai T, Harig JM, Gewertz BL 1994 Neutrophil depletion attenuates human intestinal reperfusion injury. J Surg Res 57:192-196

11. Hernandez LA, Grisham MB, Twohig B, Arfors KE, Harlan JM, Granger DN 1987 Role of neutrophils in ischemia-reperfusion-induced microvascular injury. Am J Physiol 253:H699-H703

12. Pierro A, Eaton S 2004 Intestinal ischemia reperfusion injury and multisystem organ failure. Semin Pediatr Surg 13:11-17

13. Stefanutti G, Lister P, Smith VV, Peters MJ, Klein NJ, Pierro A, Eaton S 2005 P-Selectin expression, neutrophil infiltration and histological injury in neonates with necrotizing enterocolitis. J Pediatr Surg 40:942-948

14. Zhang M, Austen WG Jr, Chiu I, Alicot EM, Hung R, Ma M, Verna N, Xu M, Hechtman HB, Moore FD Jr, Carroll MC 2004 Identification of a specific selfreactive IgM antibody that initiates intestinal ischemia/reperfusion injury. Proc Natl Acad Sci USA 101:3886-3891

15. Chen Y, Lui VC, Rooijen NV, Tam PK 2004 Depletion of intestinal resident macrophages prevents ischaemia reperfusion injury in gut. Gut 53:1772-1780

16. Cuzzocrea S, Riley DP, Caputi AP, Salvemini D 2001 Antioxidant therapy: a new pharmacological approach in shock, inflammation, and ischemia/reperfusion injury. Pharmacol Rev 53:135-159

17. Szabo C, Ohshima H 1997 DNA damage induced by peroxynitrite: Subsequent biological effects. Nitric Oxide 1:373-385

18. Rubbo H, Radi R, Trujillo M, Telleri R, Kalyanaraman B, Barnes S, Kirk M, Freeman BA 1994 Nitric oxide regulation of superoxide and peroxynitrite-dependent lipid peroxidation. Formation of novel nitrogen-containing oxidized lipid derivatives. J Biol Chem 269:26066-26075

19. Fukumoto K, Pierro A, Zammit VA, Spitz L, Eaton S 2004 Tyrosine nitration of carnitine palmitoyl transferase I during endotoxaemia in suckling rats. Biochim Biophys Acta 1683:1-6

20. Castro L, Rodriguez M, Radi R 1994 Aconitase is readily inactivated by peroxynitrite, but not by its precursor, nitric oxide. J Biol Chem 269:29409-29415

21. Salvemini D, Riley DP, Lennon PJ, Wang ZQ, Currie MG, MacArthur H, Misko TP 1999 Protective effects of a superoxide dismutase mimetic and peroxynitrite decomposition catalysts in endotoxin-induced intestinal damage. Br J Pharmacol 127:685692

22. Thiyagarajan M, Kaul CL, Sharma SS 2004 Neuroprotective efficacy and therapeutic time window of peroxynitrite decomposition catalysts in focal cerebral ischemia in rats. Br J Pharmacol 142:899-911

23. Kang JL, Lee HS, Pack IS, Leonard S, Castranova V 2001 Iron tetrakis (n-methyl$4^{\prime}$-pyridyl) porphyrinato (FeTMPyP) is a potent scavenging antioxidant and an inhibitor of stimulant-induced NF-kappaB activation of raw 264.7 macrophages. J Toxicol Environ Health A 64:291-310

24. Stefanutti G, Vejchapipat P, Williams SR, Pierro A, Eaton S 2004 Heart energy metabolism after intestinal ischaemia and reperfusion. J Pediatr Surg 39:179-183

25. Bertin R, Demarco F, Mouroux I, Portet R 1993 Postnatal-development of nonshivering thermogenesis in rats - effects of rearing temperature. J Dev Physiol 19:9-15

26. Chiu CJ, McArdle AH, Brown R, Scott HJ, Gurd FN 1970 Intestinal mucosal lesion in low-flow states. I. A morphological, hemodynamic, and metabolic reappraisal. Arch Surg 101:478-483

27. Vinardi S, Pierro A, Parkinson EJ, Vejchapipat P, Stefanutti G, Spitz L, Eaton S 2003 Hypothermia throughout intestinal ischaemia-reperfusion injury attenuates lung neutrophil infiltration. J Pediatr Surg 38:88-91

28. Stefanutti G, Pierro A, Vinardi S, Spitz L, Eaton S 2005 Moderate hypothermia protects against systemic oxidative stress in intestinal ischaemia and reperfusion injury. Shock 24:159-164

29. Horie Y, Wolf R, Miyasaka M, Anderson DC, Granger DN 1996 Leukocyte adhesion and hepatic microvascular responses to intestinal ischemia/reperfusion in rats. Gastroenterology 111:666-673

30. Naito Y, Takagi T, Ichikawa H, Tomatsuri N, Kuroda M, Isozaki Y, Katada K, Uchiyama K, Kokura S, Yoshida N, Okanoue T, Yoshikawa T 2004 A novel potent 
inhibitor of inducible nitric oxide inhibitor, ONO-1714, reduces intestinal ischemiareperfusion injury in rats. Nitric Oxide 10:170-177

31. Di Napoli M, Papa F 2005 M-40403 metaphore pharmaceuticals. IDrugs 8:67-76

32. Cuzzocrea S, Mazzon E, Dugo L, Caputi AP, Aston K, Riley DP, Salvemini D 2001 Protective effects of a new stable, highly active SOD mimetic, M40401 in splanchnic artery occlusion and reperfusion. Br J Pharmacol 132:19-29

33. Gibson DD, Brackett DJ, Squires RA, Balla AK, Lerner MR, Mccay PB, Pennington LR 1993 Evidence that the large loss of glutathione observed in ischemia reperfusion of the small-intestine is not due to oxidation to glutathione disulfide. Free Radic Biol Med 14:427-433

34. Srivastava SK, Beutler E 1969 The transport of oxidized glutathione from human erythrocytes. J Biol Chem 244:9-16
35. Schrammel A, Gorren AC, Schmidt K, Pfeiffer S, Mayer B 2003 S-nitrosation of glutathione by nitric oxide, peroxynitrite, and - $\mathrm{NO} / \mathrm{O} 2-$. Free Radic Biol Med 34:1078-1088

36. Ghezzi P 2005 Regulation of protein function by glutathionylation. Free Radic Res 39:573-580

37. Williams JP, Pechet TT, Weiser MR, Reid R, Kobzik L, Moore FD Jr, Carroll MC, Hechtman HB 1999 Intestinal reperfusion injury is mediated by IgM and complement. J Appl Physiol 86:938-942

38. Zhu L, Gunn C, Beckman JS 1992 Bactericidal activity of peroxynitrite. Arch Biochem Biophys 298:452-457

39. Ferdinandy P 2006 Peroxynitrite: just an oxidative/nitrosative stressor or a physiological regulator as well? Br J Pharmacol 148:1-3 